The most likely explanation, therefore, is that infection with the HTLV-I virus results in increased autoreactivity and autoimmune disease, a situation well described in HIV infected individuals. The increased incidence of Graves' disease, a known autoimmune disease, reported in this paper and by others, supports this view. The favourable clinical response to systemic steroids also suggests involvement of the immune system, though it would be prudent to remember that favourable responses can be seen in some infective forms of uveitis - for example, Propionibacterium acnes endophthalmitis following cataract surgery, and with ocular lymphomas. In acute retinal necrosis, now known to be caused by herpes simplex virus or herpes zoster virus in normal individuals, the visual prognosis has not been found to be improved by early intensive administration of high dose acyclovir, and immunosuppression is necessary in some patients indicating that, even in known viral retinal infection, immunological responses play a significant role in the resulting tissue damage.

To date, idiopathic panuveitis has not been a major problem in patients with HIV infection and much of the evidence for increased systemic autoimmunity is based on increased detection of autoantibodies to various tissue antigens in the blood rather than on the development of clinical disease. Dalgleish has made the interesting suggestion that if HIV could mimic major histocompatibility antigens on the surface of antigen-presenting cells, autoreactive T lymphocytes may be stimulated which would help explain the marked similarity of some of the clinical features of HIV infection to chronic graft versus host disease. ${ }^{2}$ This hypothesis could also apply to infection with other retroviruses such as HTLV-I.

If the immune system does become hyperactive as a result of retroviral infection, does direct sensitisation to ocular antigens occur resulting in the ocular inflammation? Current theories of autoimmunity suggest that $T$ cells respond to specific epitopes on infective agents which in some situations have been found to crossreact with epitopes in specific tissues. Tissue damage occurs as a consequence of this crossreactivity when these $T$ cells become activated. In addition, $\mathrm{CD}^{+} \mathrm{T}$ cells infected with HTLV-I are known to express interleukin- 2 receptors and to secrete a variety of lymphokines. ${ }^{3}$ The uveitis could then be explained by a similarity between epitopes on the HTLV-I virus and ocular tissue antigens - a sort of epiphenomenon or bystander effect occurring as a result of the activation and proliferative responses of the host's $\mathrm{T}$ cells to combat the virus.

Mochizuki et al studied the inflammatory cells in the anterior chamber using polymerase chain reaction techniques and oligonucleotide primers specific for the proviral DNA of HTLV-I in these patients. These findings were compared with two seropositive patients with other types of uveitis such as toxoplasmosis or Behçet's disease and seronegative patients with uveitis associated with sarcoidosis, Vogt-KoyanagiHarada syndrome, or Behçet's disease. The viral DNA was only detected in anterior chamber cells of seropositive patients and only in those without features of other specific types of uveitis, although there were only two patients in this group. This is a very interesting finding and further studies with increased numbers of seropositive patients with all types of uveitis are awaited. It is not known whether the cells present in the aqueous in patients with panuveitis are exactly the same in different types of uveitis or whether they also reflect those present in the retina and choroid, both in their type and specificity. These are obviously important facts to be elucidated if the HTLV-I virus is to be implicated in the pathogenesis of this ocular inflammation.

Moorfields Eye Hospital,
City Road, London ECIV 2PD

SUSAN LIGHTMAN

1 Mochizuki M, Watanabe T, Yamaguchi K, Yoshimura K, Nakashima S, Shirao M, et al. Uveitis associated with human T-cell lymphotropic virus type 1. Am F Ophthalmol 1992; 114: 123-9.

2 Dalgleish A. The pathogenesis of AIDS: classical and alternative views. $\mathcal{f}$ Roy Coll Physicians 1992; 26: 152-8.

3 Salahuddin S, Markham P, Lidner S, Popovic M, Hemmi H, Sarin S, et al. Lymphokine production by cultured human $T$-cells transformed by human T-cell leukaemia-lymphoma virus-1. Science 1984; 223: 703-7.

\title{
Measurement of flare
}

Tyndall described the light scattering effect of particles in air in 1870, as it happens, in the first volume of Nature, and the following year Lord Rayleigh worked out the mathematical principles involved describing what is now known as Rayleigh's law and explaining, for instance, why the sky is blue. Analysis of light scattering has become a routine tool in astrophysics and it is intriguing now that the same physical principles are being used to analyse the intraocular contents.

Damaged ocular blood vessels leak cells and protein whether from trauma, infection, surgery, or inflammation, and the detection of these is a part of any routine ophthalmic examination. Quantification of these signs, however, is difficult. Clinical grading scores of flare and cells are routinely used but these are subjective. Nevertheless they have stood the test of time and are still the technical standard required by the American Food and Drug Administration for the assessment of ocular inflammation. Fluorophotometry has been used to obtain more objective results but this method has never really been successful because of its invasive nature, poor patient compliance, complex pharmacokinetics, and inability to follow dynamic changes. ${ }^{12}$

Laser photometry has been developed over the last $4-5$ years by Kowa who has produced a commercially available instrument that measures flare and cells in the anterior chamber by their light scattering effects on a $\mathrm{HeNe}$ laser beam. The principle of this technique is that there is a linear relationship between protein concentration and the intensity of scattered light. ${ }^{34}$ The equipment has considerable advantages in that it is entirely non-invasive and requires minimal patient cooperation; rapid changes in the anterior chamber can be followed over minutes. With this equipment flare measurements are extremely accurate - for example, diurnal fluctuations can be measured in the normal eye. ${ }^{5}$ The sampling volume is extremely small $\left(1 / 120 \times 10^{6} / 1\right)$ and because of this, cell counts are not particularly accurate unless the numbers are high, owing to the partial volume effect. Although the 
machine is optimised to count leucocytes it will also count pigment granules and red cells which is an important consideration in the postoperative eye. Cell counts from this instrument must therefore be interpreted with caution.

Protein in the anterior chamber is a mixture of various molecular weight compounds. Light scattering is proportional to the fourth power of the molecular radius so the presence of large molecules will have a disproportionate effect on the intensity of scattered light. This becomes important in eyes with damaged blood-aqueous barriers as the aqueous contains increasing concentrations of macromolecules. Initial work with laser photometry converted the flare intensity into an equivalent albumin concentration obtained from in vitro calibration with an albumin solution. This will produce an artefactually high protein concentration in eyes with high flare counts because the effect of macromolecules is ignored and, therefore, flare measurements are better expressed as the intensity of reflected light in 'photons per ms'. ${ }^{6}$ Some caution must also be exercised in comparing eyes with different pathology; thus a photon count of 100 does not necessarily imply the same protein concentrations in an eye with uveitis as in a postoperative pseudophakic eye, as the protein composition may be different. Furthermore, protein concentration is only a marker for blood-aqueous barrier damage and the relationship of the actual inflammatory mediators to this in a particular condition may be complex. With these reservations, however, laser photometry offers considerable potential in the objective assessment of anterior chamber physiology, the assessment of inflammatory drugs and biocompatibility of intraocular materials, anterior segment surgery, and uveitis.

Recent evidence shows that posterior segment conditions such as diabetic retinopathy ${ }^{7}$ or posterior uveitis ${ }^{8}$ produce subclinical changes in blood-aqueous barrier function that can potentially be used to monitor disease progression or treatment. This may occur passively from the washing or leaching out of protein from the vitreous to the anterior chamber or directly from involvement of the blood vessels in the iris or ciliary body in the pathological process; both these mechanisms may be important in vascular retinopathies but the finding of blood-aqueous barrier changes in patients with choroidal malignant melanomas would suggest that leaching is likely to be an important mechanism. ${ }^{9}$ In this issue of the journal Nguyen and Kuchle show that the blood-aqueous barrier is deficient in patients with retinal vein occlusion and that this correlates with some parameters of the disease severity. These observations offer intriguing possibilities as a method of monitoring the natural history of the disease and correlating different pathological parameters to predict its sight threatening consequences. It also potentially offers a simple way of monitoring therapeutic intervention.

Medical Eye Unit,

D J SPALTON

St Thomas's Hospital

The author is a member of the scientific advisory panel to Kowa Acculas Inc, USA.

1 Fearnley IR, Spalton DJ, Smith SE. Anterior segment fluorophotometry in acute anterior uveitis. Arch Ophthalmol 1987; 105: 1550-5.

2 Shah SM, Spalton DJ, Allen RJ, Smith SE. A comparison of the laser flare cell meter and fluorophotometry in assessment of the blood aqueous barrier. Invest Ophthalmol 1993 (in press).

3 Sawa M, Tsurimaki Y, Tsuru T, Shimizu H. New quantitative method to determine protein concentration and cell number in aqueous in vivo. $\mathcal{F} a p \mathcal{F}$ Ophthalmol 1988; 32: 132-42.

4 Shah SM, Spalton DJ, Smith SE. Measurement of aqueous cells and flare in normal eyes. Brf Ophthalmol 1991; 75: 348-52.

5 Oshika T, Araie M, Masuda K. Diurnal variation of aqueous flare in normal human eyes measured with the laser cell flare meter. Fap $\mathcal{F}$ Ophthalmol 1988; 32: 143-50.

6 Shah SM, Spalton DJ, Taylor JC. Correlations between laser flare measurements and anterior chamber protein concentrations. Invest Ophthalmol Vis Sci 1992; 33: 2878-904.

7 Schonherr U, Kuchle M, Nguyen NX, Steinhauser B, Maumann GOH. Capillary exudation in diabetic retinopathy and diabetic iridopathy. Correlation between fluorescein angiography and laser flare cell meter. Invest Ophthalmol ARVO Abstracts 1991; 3353

8 Ferguson VM, Spalton DJ. Quantification of the ocular response to treatment in posterior uveitis. Acta Ophthalmol 1993; (in press).

9 Kuchle M, Nguyen XN, Nauman GOH. Aqueous flare in eyes with choroidal malignant melanoma. Am $\mathcal{F}$ Ophthalmol 1992; 113: 207-8. 